\title{
Recurrent parent genome recovery in different populations with the introgression of Sub1 gene from a cross between MR219 and Swarna-Sub1
}

\begin{abstract}
Recurrent parent genome (RPG) recovery is the most important step of marker assisted backcross breeding. This breeding approach is used to develop new varieties by inserting a particular gene of interest into the background of a modern variety, and removing undesirable segments linked with the target allele, which can survive after many generations of backcrossing. The marker assisted backcrossing technique is the most effective way to minimize the limitation of conventional breeding, and recover the parental genome within $2 i$ 3 generations. MR219, A modern rice variety of Malaysia (susceptible to submergence) was crossed with a high-yielding submergence tolerant variety Swarna-Sub1 (donor) to produce a new submergence tolerant rice variety. Foreground selection for the sub1 gene was done using tightly linked markers. Estimation of RPG recovery was conducted in earlier generations with previously screened polymorphic SSR markers. A total of 385 SSR markers were tested to identify the polymorphism between the parents and 88 of them found to be polymorphic. Background analysis revealed $65.55 \mathrm{i} 77.8$ and $78.79 \ddot{1} 95.5 \%$ recovery in $\mathrm{BC} 1 \mathrm{~F} 1$ and $\mathrm{BC} 2 \mathrm{~F} 1$ generations, respectively. In the $\mathrm{BC} 2 \mathrm{~F} 2$ generation the average RPG recovery was $95.37 \%$ and that for the selected plant was $96.3 \%$. This study revealed the usefulness of marker assisted backcrossing for the quick recovery of a parental genome in a backcrossing population.
\end{abstract}

Keyword: Marker assisted backcrossing; Recurrent parent genome; Rice; Sub1; Submergence 